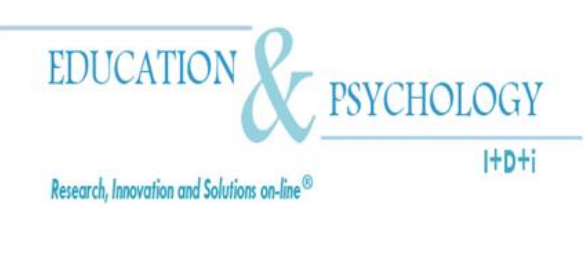

\title{
Diferencias por sexo y edad en afrontamiento infantil del estrés cotidiano
}

\section{Francisco Manuel Morales Rodríguez ${ }^{1}$, María Victoria Trianes Torres ${ }^{1}$ y Jesús Miranda Páez ${ }^{2}$}

\author{
${ }^{1}$ Departamento de Psicología Evolutiva y de la Educación, \\ Universidad de Málaga, España \\ ${ }^{2}$ Departamento de Psicobiología y Metodología de las Ciencias del \\ Comportamiento, Universidad de Málaga, España
}

\section{España}

Correspondencia: Dr. Francisco Manuel Morales Rodríguez. Departamento de Psicología Evolutiva y de la Educación. Facultad de Psicología. Universidad de Málaga. Campus de Teatinos s/n, 29071, Málaga, España. E-mail: framorrod@uma.es 


\section{Resumen}

Introducción. El estudio del afrontamiento en escolares es un tema de interés, ya que poseer habilidades de afrontamiento es garantía de vida saludable y de calidad de vida. El objetivo del presente estudio es analizar el papel que juega la edad y el sexo en la utilización de diferentes estrategias de afrontamiento ante situaciones de estrés cotidiano en escolares andaluces de educación primaria. Los problemas pertenecen a cuatro dominios de la vida diaria infantil: familiar, salud, escolar y relaciones con los iguales.

Método. Participaron 402 niños de 9-12 años. Las estrategias de afrontamiento se evaluaron con la Escala de Afrontamiento en Niños. Este instrumento consta de 35 ítems en formato de autoinforme que evalúan nueve estrategias de afrontamiento diferentes, distinguiendo entre afrontamiento centrado en el problema y afrontamiento improductivo.

Resultados. Se informa de que las niñas obtienen mayores puntuaciones estadísticamente significativas en las estrategias de afrontamiento solución activa, comunicar el problema a otros, búsqueda de información y guía y actitud positiva (afrontamiento centrado en el problema) mientras que los niños puntúan más en la estrategia conducta agresiva (afrontamiento improductivo). Respecto a la edad, los niños y niñas menores utilizan más la estrategia reservarse el problema.

Discusión y conclusiones. En este estudio se demuestra el papel relevante que juegan edad y sexo en el estudio del afrontamiento infantil en nuestro contexto, lo que tiene implicaciones directas para la evaluación e intervención psicoeducativa sobre el ámbito del estrés y su afrontamiento.

Palabras Clave: estrés infantil, afrontamiento, sexo, edad, escolares. 


\title{
Gender and Age Differences in How Children Cope with Daily Stress
}

\begin{abstract}
Introduction. The study of coping among students accounts for an interesting subject, as having coping skills guarantees a healthy lifestyle and quality of life. The present study aims to analyze the role played by age and gender on the coping strategies used by Andalusian school students to cope with situations of daily stress. These situations have been extracted from four areas in children's daily life: family, health, school and peer interaction.

Method. Participants were 402 students aged 9 to 12 years-old. Coping strategies were measured with the Childhood Coping Scale. This instrument comprises 35 self-report items that assess nine different coping strategies, distinguishing between problem-focused and nonproductive coping.
\end{abstract}

Results. Results show that girls scored higher on the coping strategies: "active solution," "telling the problem to someone else," "information and guide seeking" and "positive attitude" (problem-focused coping); whereas boys scored higher on "aggressive behaviour" (unproductive coping). As for the age factor, the youngest girls and boys show "keeping the problem to oneself" more frequently.

Discussion and Conclusion. The present study sets out to prove the relevance of age and gender in the study of children's coping in daily situations, which has got direct implications on the assessment and psycho-educational intervention on the area of stress and coping.

Keywords: children's stress, coping, gender, age, school students. 


\section{Introducción}

Las estrategias de afrontamiento son definidas como esfuerzos conscientes y voluntarios para regular emociones, conductas, cogniciones, psicofisiología y variables del entorno en respuesta al estrés de acontecimientos diarios o circunstancias (Compas, Connor-Smith, Saltzman, Thomsen y Wadsworth, 2001; Frydenberg y Lewis, 1993a). Estos recursos implican dos dimensiones de la persona, la interna, ligada a variables disposicionales, herencia, edad y sexo, y la externa, asociada a acciones ante demandas de la situación específica o el entorno sociocultural. Ambas dimensiones interactúan predisponiendo a los sujetos a comportamientos concretos y característicos (Zeidner y Saklofske, 1996).

Las habilidades de afrontamiento son consideradas un elemento influyente en la promoción de desarrollo en la infancia y adolescencia ya que las estrategias de afrontamiento utilizadas por escolares en una situación específica se relacionan con una mayor o menor adaptación y grado de bienestar psicológico (Cappa, Moreland, Conger, Dumas y Conger, 2011; González, Montoya, Casullo y Bernabéu, 2002). Como capacidades adaptativas, son eficaces frente al estrés diario reduciendo la posibilidad de enfermedad e incrementando la probabilidad de alcanzar y mantener mayores niveles de salud y calidad de vida (Folkman y Moskowitz, 2004; Urzúa y Jarne, 2008). También se demuestra que estrategias improductivas o desadaptativas se asocian a desajuste emocional, incluyendo síntomas de ansiedad y depresión (Lapointe y Marcotte, 2000; Roberts, Roberts y Chen, 2000; Seiffge-Krenke, 2000; Wright, Banerjee, Hoek, Rieffe y Novin, 2010). En otros estudios (Martínez-Monteagudo, Inglés, Trianes y García-Fernández, 2011; Sánchez, Rivas y Trianes, 2006) se señala que un clima social adecuado tiene efectos positivos sobre el ajuste psicológico, en la adaptación social y escolar así como en la capacidad de afrontamiento de niños y adolescentes.

Existe un amplio consenso para aceptar que existe un amplio factor relativo a las estrategias de afrontamiento que se denomina producción o acercamiento al problema que implica que el niño o niña se mueve para resolver el problema directamente o buscando ayudas así como también existe un amplio consenso en torno al factor improductivo o disfuncional, el cual agrupa otros afrontamientos menos efectivos o inadaptativos tales como escapar de la situación, evadirse, agredir o emitir una respuesta emocional.

Numerosos factores inciden en las estrategias de afrontamiento que utiliza un niño o niña, en concreto, la edad y el sexo, que son aspectos susceptibles de influir en las relaciones 
entre variables de riesgo y consecuencias en psicopatología (Hampel y Petermann, 2005; Lapointe y Marcotte, 2000), así como también influyen sobre la calidad del afrontamiento que se emite ante un problema o situación (Kelly, Tyrka, Price y Carpenter, 2008).

En cuanto al sexo, se consideran resultados consolidados los que encuentran que las niñas utilizan más que los niños el afrontamiento centrado en el problema, que incluye estrategias como búsqueda del apoyo social centrándose en las relaciones interpersonales además de que expresan más sus sentimientos negativos (Frydenberg y Lewis, 1993b; Patterson y McCubbin, 1987; Rhode, Lewinson, Tilson y Seeley, 1990; Seiffge-Krenke, 2007) así como parecen ser más vulnerables al estrés social (Frydenberg, 1997; SeiffgeKrenke, 2000).

Con respecto a la edad, existen también resultados que demuestran que los adolescentes utilizan mayor cantidad de estrategias de coping, comparados con niños pequeños (Eisenberg, Fabes y Guthjrie, 1997), así como más variedad de estrategias y métodos más directos que incluyen componentes cognitivos (como solución activa del problema) comparados con los más jóvenes (Williams y McGillicuddy-De Lisi, 2000). También, con la edad aumenta el uso de estrategias de afrontamiento improductivo y orientadas a la expresión de emociones (Frydenberg y Lewis, 2000). Sin embargo, existen datos contradictorios, como estudios que encuentran que el empleo de estrategias de afrontamiento centradas en el problema se adquiere al inicio de la adolescencia y se mantiene constante a lo largo de la misma (Compas, Orosan y Grant, 1993; Plancherel y Bolognini, 1995) y como estudios que no encuentran un papel claro para la edad (Dusek y Danko, 1994; Hampel y Petermann, 2005; Stern y Zevron, 1990). En relación con estos resultados inconsistentes Stone, Greenberg, Kennedy-Moore y Newman (1991), y Compas et al., (2001), entre otros, achacan al área de estudio del afrontamiento infantil una cierta dispersión debida al empleo de distintas tipologías de estrategias. En nuestro caso se evalúan por medio de un autoinforme que presenta cuatro dominios de estresores potenciales en niños y preadolescentes con una tipología de afrontamientos consolidada en la literatura. 


\section{Objetivos e hipótesis}

El objetivo principal del presente estudio es establecer la existencia de diferencias en función del sexo y la edad en la Escala de Afrontamiento Infantil (EAN), que aporta utilidades para el tratamiento del estrés, tanto en el ámbito clínico como educativo. Los objetivos específicos son: (1) Analizar diferencias en el tipo de estrategia de afrontamiento utilizada en función del sexo comprobando si los niños utilizan un tipo de estrategia de afrontamiento diferente a las niñas; y (2) Examinar si existen diferencias en el tipo de estrategia de afrontamiento utilizada en función de la edad, analizando también la interacción edad x sexo.

\section{Método}

\section{Participantes}

Se trabajó con una muestra incidental de 402 sujetos, 190 niñas (47.5\%) y 212 niños (52.4\%) con edades comprendidas entre los 9 y 12 años, pertenecientes a dos colegios públicos y dos colegios concertados de Málaga con una media de 10.49 años $(D T=1.00)$. Puede observarse en la Tabla 1 que la composición de la muestra está equilibrada según el sexo y que la mayoría de los participantes tienen edades comprendidas entre los 10 y 11 años, pudiéndose hablar de infancia tardía e incluso preadolescencia. Los centros educativos corresponden a zonas urbanas con familias de un nivel socioeconómico medio.

Tabla 1. Distribución de la muestra según el sexo y la edad de los participantes

\begin{tabular}{lllll} 
& Niña & & Niño \\
\hline Edad & Frecuencia & Porcentaje & Frecuencia & Porcentaje \\
\hline 9 años & 37 & $45.7 \%$ & 44 & $54.3 \%$ \\
10 años & 61 & $48.8 \%$ & 64 & $51.2 \%$ \\
11 años & 63 & $45.9 \%$ & 74 & $54.0 \%$ \\
12 años & 30 & $50.8 \%$ & 29 & $49.1 \%$ \\
\hline
\end{tabular}

\section{Instrumento}

Escala de afrontamiento en niños, EAN (Morales et al., 2011). Para evaluar las estrategias de afrontamiento utilizada por los escolares se utiliza el inventario EAN que recoge estrategias de afrontamiento seleccionadas por expertos como representativas del área del afrontamiento infantil. Este instrumento autoinformado chequea estrategias de afrontamiento suscitadas ante cuatro problemas, relacionado con el contexto familiar, la salud, las tareas escolares, y las relaciones sociales. Consta de 35 ítems con un formato de respuesta en escala 
tipo Likert de 3 puntos (nunca, algunas veces y muy frecuentemente), que evalúa estrategias de afrontamiento diferentes, distinguiendo dos factores generales: afrontamiento centrado en el problema y afrontamiento improductivo. El afrontamiento centrado en el problema está formado por las siguientes estrategias: solución activa, comunicar el problema a otros, búsqueda de información y guía, y actitud positiva; y el factor afrontamiento improductivo incluye estas estrategias: indiferencia, conducta agresiva, reservarse el problema para sí mismo, evitación cognitiva y evitación conductual.

Este instrumento presenta adecuadas propiedades psicométricas, mostrando un buen ajuste y saturaciones significativas, oscilando los índices de fiabilidad (coeficiente alpha de Cronbach) para cada uno de los factores/estrategias evaluadas en la presente muestra entre .52 y .85 .

\section{Procedimiento}

Se contactó con los distintos centros de Educación Primaria informándoles por escrito de los objetivos del proyecto, al mismo tiempo que se solicitó a los padres su autorización y conformidad en la investigación, proporcionándoles las correspondientes instrucciones y notas informativas. Para la administración de las diversas pruebas se informó a los alumnos sobre el carácter voluntario de la participación, garantizando en todo momento la confidencialidad de los datos, mediante su tratamiento global. El estudio fue aprobado por el correspondiente Comité de Ética de la institución que llevó a cabo la investigación.

La aplicación fue desarrollada por dos investigadores entrenados tanto para explicar los objetivos al profesorado como para dar las instrucciones adecuadas al alumnado. Los cuestionarios se pasaron de forma colectiva en las distintas clases de Educación Primaria.

\section{Análisis Estadístico}

Para la organización y el análisis de los datos se ha utilizado el paquete estadístico informatizado SPSS V.17. Se han realizado los siguientes análisis: Análisis descriptivos uni y bivariados para conocer la forma en que se distribuyen las variables en la muestra. Para analizar diferencias en función de la edad y sexo se han aplicado análisis simples de la varianza/análisis de varianza univariados, ANOVA y con el objetivo de determinar el efecto cruzado de estas variables se realiza un análisis múltiple de la varianza/análisis multivariado, MANOVA. Las VDs la forman los factores/estrategias de afrontamiento (afrontamiento centrado en el problema: solución activa, comunicar el problema a otros, búsqueda de información y 
guía, y actitud positiva; y afrontamiento improductivo: indiferencia, conducta agresiva, reservarse el problema para sí mismo, evitación cognitiva, y evitación conductual). Los análisis post hoc (prueba de subconjuntos homogéneos) confirman las diferencias entre estas agrupaciones de estrategias así como la conveniencia misma de dicha clasificación. Las VIs asignadas son edad y sexo.

\section{Resultados}

A continuación en la Tabla 2 se muestran los descriptivos de las estrategias (media y desviación típica) del total de la muestra en función del sexo y de la edad. Se desprende que en esta muestra predomina la estrategia solución activa que es más utilizada por las niñas de 12 años.

Tabla 2. Media y desviación típica (entre paréntesis) en cada estrategia en función del sexo y de la edad

\begin{tabular}{|c|c|c|c|c|c|c|c|c|}
\hline \multirow{4}{*}{ Sexo } & \multicolumn{8}{|c|}{ Edad } \\
\hline & \multicolumn{2}{|c|}{9} & \multicolumn{2}{|c|}{10} & \multicolumn{2}{|c|}{11} & \multicolumn{2}{|c|}{12} \\
\hline & Niña & Niño & Niña & Niño & Niña & Niño & Niña & Niño \\
\hline & Media & Media & Media & Media & Media & Media & Media & Media \\
\hline Indiferencia & 6.2 & 6.0 & 5.5 & 6.2 & 5.3 & 6.3 & 5.3 & 6.1 \\
\hline & (1.3) & $(1.5)$ & $(1.0)$ & $(1.6)$ & (1.4) & (1.8) & $(1.3)$ & (1.9) \\
\hline Conducta agre- & 5.1 & 5.6 & 4.6 & 5.4 & 5.3 & 5.6 & 4.8 & 6.0 \\
\hline siva & (1.6) & $(2.0)$ & $(.98)$ & (1.4) & (1.6) & (1.6) & $(1.2)$ & $(1.7)$ \\
\hline Reservarse el & 6.8 & 7.0 & 6.4 & 6.3 & 6.2 & 6.5 & 5.6 & 6.4 \\
\hline problema & (1.9) & $(2.05)$ & (1.7) & (1.8) & (2.0) & (2.0) & $(1.2)$ & (1.8) \\
\hline Evitación cog- & 7.1 & 7.2 & 6.8 & 7.1 & 7.1 & 7.4 & 7.7 & 7.6 \\
\hline nitiva & (1.6) & (1.9) & (1.7) & (1.7) & (1.6) & (1.9) & $(1.5)$ & (1.5) \\
\hline Evitación con- & 6.7 & 6.4 & 6.3 & 6.7 & 6.7 & 7.0 & 7.4 & 6.7 \\
\hline ductual & (1.6) & (1.9) & (1.6) & (1.5) & (1.8) & (2.0) & $(1.7)$ & (1.7) \\
\hline Solución activa & 10.5 & 10.1 & 10.5 & 10.1 & 10.5 & 10.1 & 10.7 & 9.3 \\
\hline & $(1.6)$ & (1.8) & (1.4) & (1.8) & (1.5) & (1.8) & $(1.4)$ & $(1.8)$ \\
\hline Comunicar el & 8.3 & 8.2 & 7.7 & 7.2 & 8.5 & 7.5 & 8.5 & 7.7 \\
\hline $\begin{array}{l}\text { problema a } \\
\text { otros }\end{array}$ & $(1.6)$ & $(2.1)$ & $(2.1)$ & $(2.0)$ & (1.8) & $(2.0)$ & $(1.8)$ & $(2.4)$ \\
\hline Búsqueda de & 8.6 & 7.5 & 7.9 & 7.6 & 8.4 & 7.6 & 8.0 & 7.8 \\
\hline $\begin{array}{l}\text { información y } \\
\text { guía }\end{array}$ & $(2.0)$ & $(1.9)$ & $(1.8)$ & $(2.1)$ & (2.3) & (1.9) & $(1.8)$ & (1.7) \\
\hline Actitud positiva & 9.5 & 9.1 & 9.8 & 9.0 & 10 & 9.5 & 10.1 & 9.2 \\
\hline & $(2.0)$ & $(2.3)$ & $(1.8)$ & $(2.1)$ & (1.6) & (2.0) & $(1.7)$ & $(2.1)$ \\
\hline Afrontamiento & 32.1 & 32.3 & 29.8 & 31.7 & 30.7 & 32.8 & 30.8 & 32.8 \\
\hline improductivo & $(5.2)$ & (7.1) & $(4.1)$ & $(6.2)$ & $(5.9)$ & $(6.8)$ & $(4.8)$ & $(6.5)$ \\
\hline Afrontamiento & 37.1 & 35.0 & 36.0 & 34.0 & 37.3 & 34.7 & 37.3 & 34.1 \\
\hline $\begin{array}{l}\text { centrado en el } \\
\text { problema }\end{array}$ & $(4.9)$ & $(6.2)$ & (4.7) & (6.1) & $(5.5)$ & $(5.6)$ & $(4.6)$ & $(6.7)$ \\
\hline
\end{tabular}

Todas las estrategias de afrontamiento presentan una distribución diferencial en función del sexo, como se observa en la Tabla 3 a partir de los análisis de la varianza 
(ANOVA), excepto en el caso de las estrategias de evitación tanto conductual como cognitiva y reservarse el problema para sí, en las que en esta muestra no se aprecian diferencias estadísticamente significativas en función de esta variable. Los resultados demuestran, por tanto, que las niñas presentan un uso más elevado de todas las estrategias de afrontamiento centrado en el problema analizadas: solución activa, comunicar el problema a otros, búsqueda de información y guía, y actitud positiva.

Para la estrategia indiferencia (afrontamiento improductivo) también existen diferencias estadísticamente significativas en función del sexo. Esta estrategia es más empleada por las niñas de 9 años, aunque su empleo es mayor por los niños del resto de las edades (10, 11 y 12 años). Asimismo para la estrategia de afrontamiento improductivo conducta agresiva la puntuación media es mayor para los niños.

En relación a las diferencias estratégicas en función de la edad, solo se aprecian diferencias claras en el caso de la estrategia reservarse el problema. Los resultados obtenidos indican, como se muestra en la Figura 1, que a menor edad mayor es el empleo de la estrategia reservarse el problema en ambos sexos. Esta disminución a mayor edad en el empleo de dicha estrategia es estadísticamente significativa $(\mathrm{p}<.01)$, y es especialmente señalada o empleada por niños y niñas de 9 años.

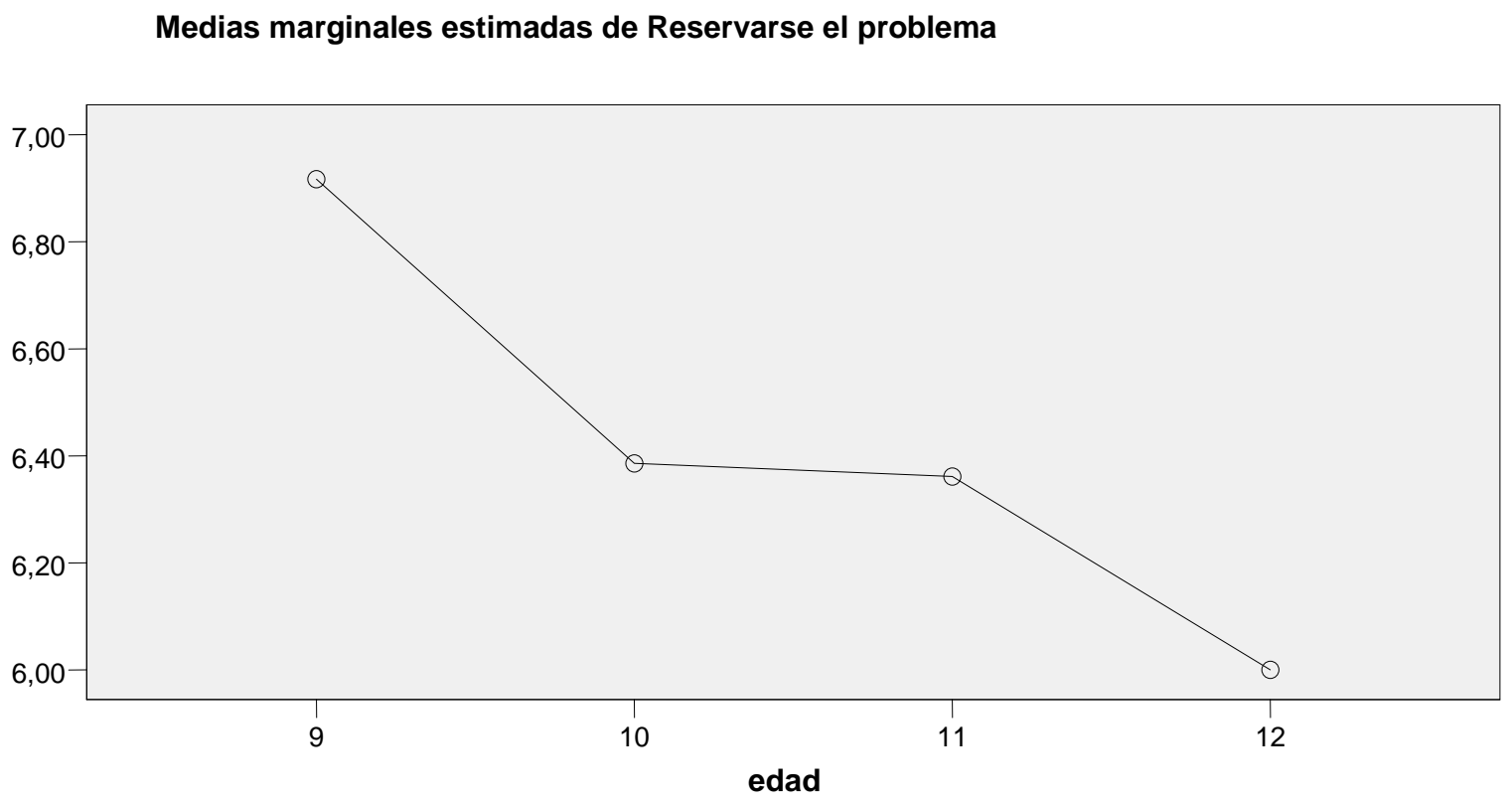

Figura 1. Medias estimadas marginales de reservarse el problema en función de la edad 
Para determinar los efectos de las interacciones sobre las estrategias de afrontamiento se realiza un análisis multivariado de la varianza. Como se observa en la Tabla 3 , los dos factores señalados, sexo y edad, tienen efectos significativos simples la mayoría al 99\% $(\mathrm{p}<.01)$ en el caso del sexo, aunque en el caso de la edad el efecto es significativo al $95 \%$ $(\mathrm{p}<.05)$ mientras que sus interacciones en esta muestra no demuestran efectos significativos estadísticamente a ninguno de esos niveles de confianza. Para las estrategias de evitación cognitiva y conductual no existen diferencias estadísticamente significativas. Para conocer la dimensión de las diferencias entre los grupos, se halló el tamaño del efecto para cada diferencia significativa. El rango de los tamaños del efecto oscilan entre .19 y .50 siendo mayores las diferencias para las niñas de mayor edad.

Tabla 3. Diferencias en las estrategias de afrontamiento en función del sexo y de la edad

\begin{tabular}{|lllllllllll|}
\hline & Sexo & \multicolumn{3}{c}{ Edad } & \multicolumn{5}{c|}{ Sexo x edad } \\
& & & & & & & & \\
& $F$ & $p$ & $\eta 2$ & $F$ & $p$ & $\eta 2$ & $F$ & $p$ & $\eta 2$ \\
Indiferencia & 11.5 & .00 & .02 & .85 & .46 & .00 & 2.1 & .10 & .01 \\
Conducta agresiva & 13.6 & .00 & .03 & 2.1 & .09 & .01 & 1.3 & .26 & .01 \\
Reservarse el problema & 1.7 & .19 & .00 & 2.8 & .04 & .02 & .72 & .54 & .01 \\
Evitación cognitiva & .44 & .50 & .00 & 1.9 & .13 & .01 & .27 & .84 & .00 \\
Evitación conductual & .05 & .81 & .00 & 1.5 & .20 & .01 & 1.5 & .21 & .01 \\
Solución activa & 13.8 & .00 & .03 & .48 & .69 & .00 & 1.3 & .27 & .01 \\
Comunicar el problema a otros & 6.0 & .01 & .01 & 2.5 & .06 & .01 & .80 & .49 & .00 \\
Búsqueda de información y guía & 7.2 & .00 & .01 & .52 & .66 & .00 & .98 & .40 & .00 \\
Actitud positiva & 8.2 & .00 & .02 & .90 & .44 & .00 & .32 & .80 & .00 \\
Afrontamiento improductivo & 5.6 & .02 & .01 & 1.0 & .37 & .00 & .48 & .69 & .00 \\
Afrontamiento centrado en el pro- & 16.4 & .00 & .04 & .83 & .48 & .00 & .18 & .90 & .00 \\
blema & & & & & & & & & \\
\hline
\end{tabular}

\section{Discusión y conclusiones}

En este estudio se ha adoptado un modelo o enfoque sobre la concepción del afrontamiento que lo entiende multicausado, es decir, dependiente de las demandas de la situación, del desarrollo del alumno, del sexo y de la edad (Goyen y Anshel, 1998) entre otros factores. Este enfoque o modelo es predominante hoy cuando se buscan comprensiones complejas de las causas de la psicopatología infantil en los que las variables estrés y afrontamiento bien como mediadoras, bien como factores de riesgo (Grant et al., 2006) están llamadas a desempeñar un importante papel. Nuestro objetivo era averiguar las diferencias por edad y sexo en las estrategias de afrontamiento ante una selección de estresores cotidianos o problemas propios de la vida diaria infantil. 
Se hallaron diferencias significativas en función del sexo en el empleo de la mayoría de las estrategias de afrontamiento, en especial, en las que engloban el afrontamiento centrado en el problema, a favor de las niñas. Concretamente, la estrategia solución activa obtiene un contraste significativo en el cual las niñas obtienen mayor puntuación. Estos datos se encuentran en consonancia con estudios actuales que informan en las niñas de un mayor esfuerzo por buscar soluciones activas al problema (González et al., 2002; Patterson y McCubbin, 1987; Seiffge-Krenke, 2007; Seiffge-Krenke y Stemmler, 2002). En cambio, en relación al afrontamiento improductivo, la tónica es que los niños de todas las edades obtienen mayor puntuación que las niñas. Otros estudios también concluyen que con la edad aumenta en los niños el uso de estrategias de afrontamiento improductivo tales como ignorar el problema y reservar el problema para sí (Frydenberg y Lewis, 2000). Otras investigaciones también obtienen resultados concordantes con el presente estudio ya que encuentran que los niños presentan mayor puntuación en afrontamiento improductivo, analizándose que obtienen mayor peso debido a dos componentes de esa dimensión que son agresión, junto con ocultación del problema e indiferencia. Así, estos resultados pueden relacionarse con otros que encuentran en los niños más afrontamiento improductivo basado la evaluación de agresión e indiferencia (Baker, 2006; Frydenberg, 1997; Prior, Smart, Sanson y Oberklaid, 1993) así como en ignorar el problema y reservarlo para sí (González et al., 2002). Otros estudios en la infancia media (Hampel y Petermann, 2005; Sandstrom, 2004) también encuentran resultados convergentes. No obstante, existen puntos de vista que afirman que las diferencias en estrategias de afrontamiento improductivas entre niños y niñas no se perciben bien en la infancia media, sino que se diferencian mejor en la adolescencia (Reijntjes, Stegge y Meerum-Terwogt, 2006).

Respecto a las estrategias evitación cognitiva y evitación conductual éstas no discriminan entre niños y niñas, resultado que parece acorde con la literatura que no encuentra un papel claro para estas estrategias (Compas et al., 2001). La variable sexo también produce diferencias significativas en el uso de las estrategias actitud positiva, comunicar el problema a otros y búsqueda de información y guía en las que las niñas obtienen mayor puntuación, resultados concordantes con otras investigaciones previas (Frydenberg y Lewis, 1993b, 2000; González et al., 2002; Hampel y Petermann, 2005; Seiffge-Krenke, 1993).

A continuación se discuten las diferencias en función de la edad, teniendo en cuenta que este factor ha arrojado diferencias a nivel principal pero no en interacción con el factor 
sexo. Concretamente la edad parece incidir de manera individual menos que el sexo y produce diferencias estadísticamente significativas en el empleo de la estrategia de afrontamiento reservarse el problema siendo más empleada por los niños de menor edad (9 años). Algunos autores encuentran que conforme aumenta la edad a lo largo de la adolescencia el empleo de la estrategia emocional que incluye aspectos como mayor tendencia a expresar emociones y contar el problema a los demás es mayor (Frydenberg y Lewis, 1993b). En este sentido, resulta esperable que los niños de menor edad ante situaciones que les inspiran temor tiendan más a reservar u ocultar el problema para sí mismos. Finalmente es preciso señalar que, respecto a la interacción sexo $\mathrm{x}$ edad, no hay un resultado significativo en los contrastes efectuados, resultado congruente con otros estudios (Williams y McGillicuddy-De Lisi, 2000).

Como conclusión de las diferencias por sexo y edad se dibuja un perfil para la niña acorde con los nuevos cánones de socialización de género. Este perfil presenta luces y sombras. Las niñas aparecen más capaces de solución activa ante los problemas presentándose con la motivación de acometer los problemas y resolverlos. También tienen mayor puntuación en las estrategias consistentes en comunicar el problema, y búsqueda de información y guía. Este último resultado puede ser debido a que las niñas pueden informar más que los chicos de sus alteraciones emocionales sin sentirse censuradas por ello. Esto se puede explicar debido a los diferentes procesos de socialización de los dos sexos. Las niñas otorgan mayor importancia a las relaciones interpersonales en todos los procesos de socialización primaria referidos a identidad, relaciones y estereotipos de género existentes en nuestra sociedad (Crick y Grotpeter, 1995). Esto puede ayudar a explicar la mayor predisposición de las niñas a informar de sus desajustes emocionales a diferencia de los niños, que se pueden sentir, por la deseabilidad social, más inhibidos a mostrar y expresar sus emociones reales. Los diferentes procesos de socialización tanto primaria como secundaria existentes en ambos sexos, pueden llevar a una mayor permisividad en la expresión de emociones en las niñas (Hampel y Petermann, 2005; Kouzma y Kennedy, 2002).

Para terminar cabe enfatizar que ha quedado demostrado en este estudio el papel relevante que juegan edad y sexo en el estudio del afrontamiento infantil lo que tiene implicaciones directas para la evaluación e intervención psicoeducativa sobre el ámbito del estrés y su afrontamiento. No puede pensarse esta evaluación e intervención psicoeducativa sin considerar el impacto de estas dos variables, que están llamadas a desempeñar papeles de 
variables mediadoras o moderadoras en enfoques complejos multidimensionales y multivariados.

Entre las posibles limitaciones que presenta este estudio se encuentra, por ejemplo, la recomendación de seguir empleando en futuros estudios análisis multivariados más robustos que permitan llegar a conclusiones más precisas y con mayor certeza. En futuras investigaciones también sería preciso controlar el estilo parental de crianza empleado en niños y niñas. A pesar de estas limitaciones, los hallazgos de este estudio resultan relevantes para el desarrollo de acciones preventivas e intervenciones psicoeducativas más eficaces sobre el afrontamiento del estrés cotidiano infantil, ayudando a diseñar mejores tratamientos. Estas implicaciones conllevan un enfoque de prevención primaria ante situaciones de estrés cotidiano en escolares a través de programas y actividades educativas dirigidos tanto al manejo del estrés como al empleo de estrategias de afrontamiento más productivas y eficaces por parte de los mismos.

\section{Agradecimientos}

Agradecemos a los revisores las sugerencias realizadas para la mejora de este trabajo.

\section{Referencias}

Baker, J. A. (2006). Contributions of teacher-child relationships to positive school adjustment during elementary school. Journal of School Psychology, 44, 211-229.

Cappa, K., Moreland, A., Conger, J., Dumas, J. y Conger, A. (2011). Bidirectional relationships between parenting stress and child coping competence: Findings from the pace study. Journal of Child and Family Studies, 20, 334-342.

Compas, B. E., Connor-Smith, J. K., Saltzman, H., Thomsen, A. H. y Wadsworth M. E. (2001). Coping with stress during chilhood and adolescence: Problems, progress, and potencial in theory and research. Psychological Bulletin, 127, 87-127.

Compas, B. E., Orosan, P. G. y Grant, K. E. (1993). Adolescent stress and coping: Implications for psychopathology during adolescence. Journal of Adolescence, 16, 331-349.

Crick, N. R. y Grotpeter, J. K. (1995). Relational agression, gender and social-psychological adjustment. Child Development, 66, 710-722.

Dusek, I. B. y Danko, M. (1994). Adolescent coping styles and perceptions of parental child rearing. Journal of Adolescent Research, 9, 412-426.

Eisenberg, N., Fabes, R. A. y Guthjrie, I. K. (1997). Coping with stress: The roles of regula- 
tion and development. En S. A. Wolchik y I. N. Sandler (Eds.), The handbook of children's coping (pp. 41-70). New York, NY: Plenum.

Folkman, S. y Moskowitz, J. T. (2004). Coping: pitfalls and promise. Annual Review of Psychology, 55, 745-774.

Frydenberg, E. (1997). Adolescent coping. Theoretical and research perspectives. New York, NY: Routledge.

Frydenberg, E., y Lewis, R. (1993a). The Adolescent Coping Scale: Practitioners manual. Melbourne: Australian Council for Educational Research.

Frydenberg, E. y Lewis, R. (1993b). Boys play sport and girls turn to others: Age, gender and ethnicity as determinants of coping. Journal of Adolescence, 16, 253-266.

Frydenberg, E. y Lewis, R. (2000). Coping with stresses and concerns during adolescence: A longitudinal study. American Educational Research Journal, 37, 727-745.

González, R., Montoya, I., Casullo, M. y Bernabéu, J. (2002). Relación entre estilos y estrategias de afrontamiento y bienestar psicológico en adolescentes. Psicothema, 14, 363368 .

Goyen, M. y Anshel, M. (1998). Sources of acute competitive stress and use of coping strategies as a function of age and gender. Journal of Applied Developmental Psychology, 19, 469-486.

Grant, K. E., Compas, B. E., Thurm, A. E., McMahon, S. D., Gipson, P. Y., Campbell, A. J., et al. (2006). Stressors and child and adolescent psychopathology: Evidence of moderating and mediating effects. Clinical Psychology Review, 26, 257-283.

Hampel, P. y Petermann, F. (2005). Age and gender effects on coping in children and adolescents. Journal of Youth and Adolescence, 34, 73-83.

Kelly, M., Tyrka, A., Price, L. y Carpenter, L. (2008). Sex differences in the use of coping strategies: Predictors of anxiety and depressive symptoms. Depression and Anxiety, $25,839-846$.

Kouzma, N. M. y Kennedy, G. A. (2002). Homework, stress, and mood disturbance in senior high school students. Psychological Reports, 91, 193-198.

Lapointe, V. y Marcotte, D. (2000). Gender typed characteristics and coping strategies of depressed adolescents. European Review of Applied Psychology, 50, 451-460. 
Martínez-Monteagudo, Inglés, Trianes y García-Fernández (2011). Perfiles de ansiedad escolar: Diferencias en clima social y violencia entre iguales. Electronic Journal of Research in Educational Psychology, 9, 1023-1042.

Morales, F. M., Trianes, M. V. y Blanca, M. J. (Marzo, 2011). Estrategias de afrontamiento y su relación con el ajuste psicológico y rendimiento académico en niños y adolescentes de Educación Primaria. Póster presentado al VI Congreso Internacional de Psicología y Educación y el III Congreso Nacional de Psicología de la Educación, Valladolid, España.

Morales, F. M., Trianes, M. V., Blanca, M. J., Miranda, J. M., Escobar, M. y Fernández, F. J. (2011). Escala de afrontamiento en niños: Propiedades psicométricas. Anales de Psicología, 28, 289-302.

Patterson, J. M. y McCubbin, H. I. (1987). Adolescent, coping style and behaviors: conceptualization and measurement. Journal of Adolescence, 10, 163-186.

Plancherel, B. y Bolognini, M. (1995). Coping and mental health in early adolescence. Journal of Adolescence, 18, 459-474.

Prior, M., Smart, D., Sanson, A. y Oberklaid, F. (1993). Sex differences in psychological adjustment from infancy to 8 years. Journal of the American Academy of Child and Adolescent Psychiatry, 32, 291-304.

Reijntjes, A., Stegge, H. y Meerum-Terwogt, M. (2006). Children's coping with peer rejection: The role of depressive symptoms, social competence, and gender. Infant and Child Development, 15, 89-107.

Rhode, P., Lewinson, P. M., Tilson, M. y Seeley, J. R. (1990). Dimensionality of coping and its relationship to depression. Journal of Personality and Social Psychology, 58, 499511.

Roberts, R. E., Roberts, C. R. y Chen, I. G. (2000). Fatalism and risk of adolescent depression. Psychiatry Interpersonal and Biological Processes, 63, 239-252.

Sánchez, A. M., Rivas, M. T. y Trianes, M. V. (2006). Eficacia de un programa de intervención para la mejora del clima escolar: algunos resultados. Electronic Journal of Research in Educational Psychology, 4, 353-370.

Sandstrom, M. J. (2004). Pitfalls of the peer world: How children cope with common rejection experiences. Journal of Abnormal Child Psychology, 32, 67-81. 
Seiffge-Krenke, I. (1993). Coping behavior in normal and clinical samples: More similarities that differences. Journal of Adolescence, 16, 285-303.

Seiffge-Krenke, I. (2000). Causal links between stressful events, coping style and adolescent symptomatology. Journal of Adolescence, 23, 675-691.

Seiffge-Krenke, I. (2007, Febrero). Stress, coping and depression. Ponencia presentada en el Postgrado de Investigación en Procesos Básicos e Intervención en Psicología de la Salud, bienio 2006-2008, Universidad de Málaga, España.

Seiffge-Krenke, I. y Stemmler, M. (2002). Factors contributing to gender differences in depressive symptoms: A test of three developmental models. Journal of Youth and Adolescence, 31, 405-417.

Stern, M. y Zevron, M. A. (1990). Stress, coping and family environment: The adolescent's response to naturally occurring stressors. Journal of Adolescent Research, 5, 290-305.

Stone, A. A., Greenberg, M., Kennedy-Moore, E. y Newman, M. G. (1991). Self-report, situation-specific coping questionnaires: What are they measuring? Journal of Personality and Social Psychology, 61, 648-658.

Urzúa, A. y Jarne, A. (2008). Calidad de vida y estilos de afrontamiento en personas con patologías crónicas. Interamerican Journal of Psychology, 42, 151-160.

Williams, K. y McGillicuddy-De Lisi, A. (2000). Coping strategies in adolescents. Journal of Applied Developmental Psychology, 20, 537-549.

Wright, M., Banerjee, R., Hoek, W., Rieffe, C. y Novin, S. (2010). Depression and social anxiety in children: Differential links with coping strategies. Journal of Abnormal Child Psychology, 38, 405-419.

Zeidner, M. y Saklofske, D. (1996). Adaptive and maladaptive coping. En M. Zeidner y N. E. Endler (Eds), Handbook of coping: theory, research, applications. Nueva York: Wiley. 